\title{
Traumatic arteriovenous fistula
}

\author{
GLENN E. HAAS, Do
}

\section{Traumatic arteriovenous} fistula is caused by simultaneous injury to an adjacent artery and vein. The resultant communication between the two structures may remain asymptomatic or may resolve spontaneously. As the fistula increases in size, symptoms referable to the organ or region involved develop. Local symptoms are a result of arterial insufficiency and venous hypertension. Systemic symptoms are a manifestation of high-output cardiac failure and pulmonary hypertension.

Surgical intervention is indicated prior to the development of systemic complications. The optimal procedure is primary repair of both vessels and preservation of vascular continuity. Presented are three cases that exemplify traumatic fistulas affecting various anatomic sites (brachial, coronary artery-right atrial, and renal fistulas). Each produced symptoms referable to the involved organ as well as to the cardiovascular and pulmonary systems.

Acquired arteriovenous fistula most often is caused traumatically. This simultaneous injury to an adjacent artery and vein may occur as one significant episode, or may result from repetitive episodes. Penetrating trauma (missile injuries, stabbings, shrapnel wounds) is common in either a military or civilian setting. Blunt trauma, tumor erosion, sepsis, rupture of an atherosclerotic artery into an adjacent vein, and radiation therapy all have been implicated as etiologic events.

Although the extremities are the most common sites for these fistulas (femoral, popliteal, brachial, subclavian fistulas), ${ }^{1}$ few areas of the body are absolutely protected from their development. No doubt, many persons with arteriovenous fistulas remain asymptomatic; however, ischemic symp- toms distal to the fistula are common and are referable to the organ involved. Systemic symptoms include high-output cardiovascular failure and pulmonary hypertension.

The three cases presented in this article each produced symptoms referable to the involved organ and the cardiovascular and pulmonary systems. The pathologic and physiologic effects of these injuries, as well as various methods of therapy, are discussed.

\section{Report of cases}

\section{Case 1 (brachial fistula)}

A 31-year-old man, a laborer who worked with glass and mirror panels, and sheets of plywood and plasterboard, complained of pain and weakness in his left arm. The symptoms became worse during activity. The patient, who was left-handed, had noted decreased fine motor coordination in the hand and fingers. He also had become more aware of increasing dyspnea and fatigue.

There was no significant radial pulse in the left arm. The brachial systolic pressures were decreased markedly on the left side, and there was no left diastolic component. A thrill was appreciated across the left shoulder and chest and involved the entire left arm. Two years prior to this hospitalization, the patient had undergone surgery for ulnar nerve transposition on the left elbow, with resection of the radial head. None of these findings were present at that time, or during the postoperative period. Consequently, there was no apparent association between surgery and fistula.

Angiography demonstrated a large fistula between the brachial artery and a vein at the antecubital fossa (Fig 1). The fistula was surgically divided and ligated, the brachial artery was repaired with a venous patch, and primary lateral repair of the vein was performed. The postoperative course was uneventful.

One year after surgery, the patient has full use of the arm and adequate fine motor activity of the hand. Radial pulse pressures are equal bilaterally.

Case 2 (coronary artery-right atrial fistula)

A 22-year-old man was treated in the emergency room 
for gunshot wounds to the jaw, neck, chest, and abdomen. He was in profound shock. Left thoracotomy was performed for cardiac massage and resuscitation purposes. He was transported to the operating room for repair of lacerations in the right atrial appendage and wall. Laparotomy to repair a lacerated liver and tracheostomy to repair laryngeal injuries were performed.

Postoperative electrocardiography demonstrated acute inferior wall myocardial infarction. The patient recovered uneventfully and left the hospital in satisfactory condition, but he did not return for follow-up care.

One year later, he was evaluated for exertional dyspnea and chest pain. A "machinery murmur" was noted across the precordial area. Cardiomegaly was demonstrated on the chest roegentographic film (Fig 2). His symptoms worsened and the murmur increased in intensity.

During cardiac catheterization, a right coronary arteryright atrial fistula was noted (Fig 3). Distal to the fistula, the right coronary artery was occluded and the posterior descending artery filled from the left coronary artery collateral circulation.

At subsequent surgery, the right coronary artery was dissected free. A thrill was present over the right coronary artery inferior to the atrial appendage. After cardiopulmonary bypass and potassium cardioplegia were accomplished, the right coronary artery was ligated proximally and distally to the fistula. The thrill disappeared. Aorto-right coronary artery bypass was performed using a reverse saphenous vein graft.

The postsurgical course was uneventful. When the patient was reevaluated four months later, the dyspnea and chest pain had resolved, no murmur was present, and the chest film was normal.

\section{Case 3 (renal fistula)}

A 41-year-old man who had been maintained on hemodialysis for chronic renal failure was evaluated for hema-

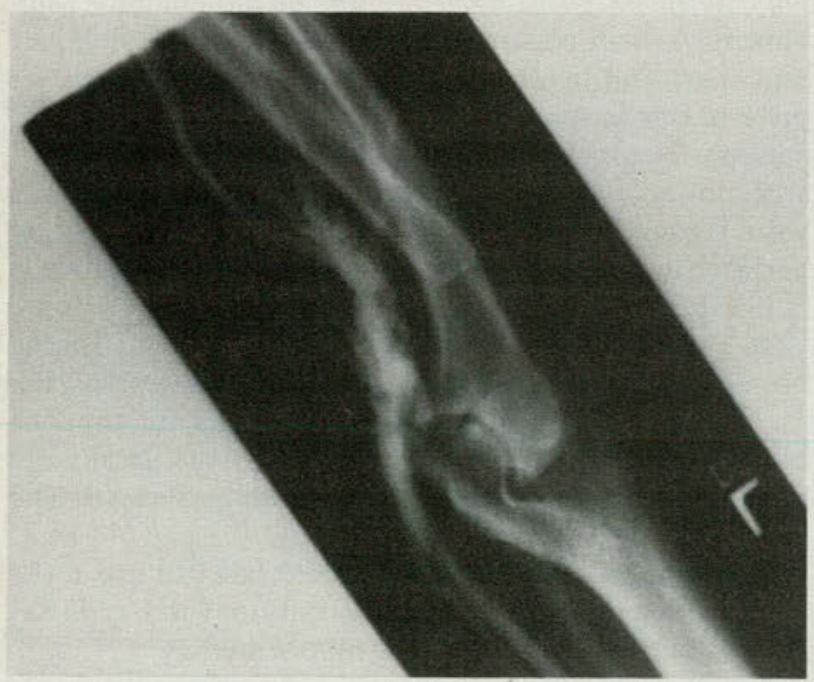

Fig. 1. Angiographic demonstration of brachial arteriovenous fistula (case 1).

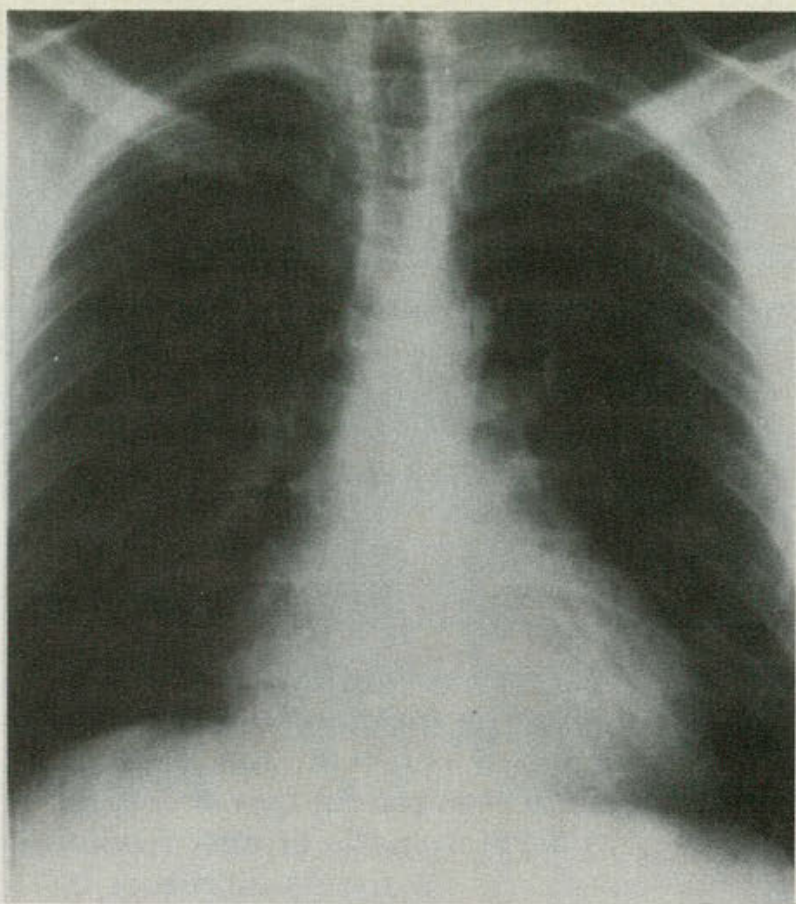

Fig 2. Chest roentgenogram shows cardiomegaly in a patient with coronary artery-right atrial fistula (case 2).

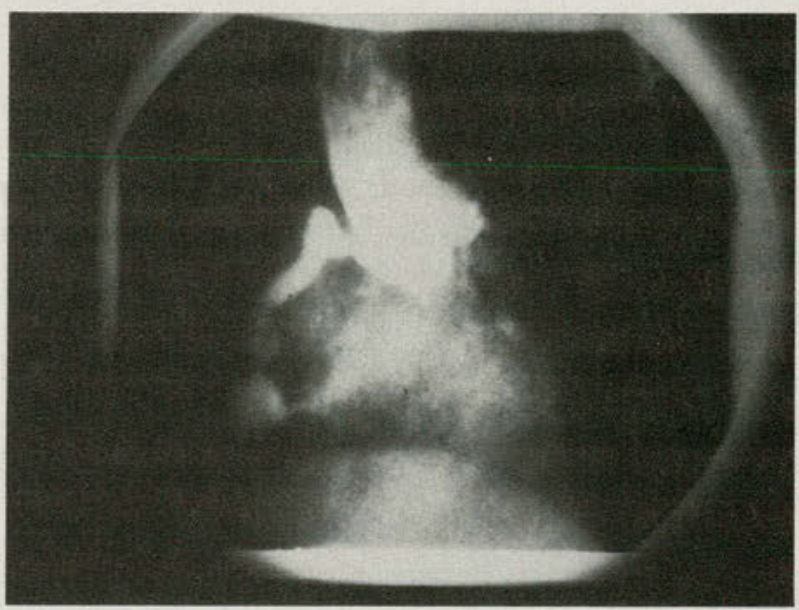

Fig 3. Cardiac catheterization demonstrates right coronary arteryright atrial fistula. Aneurysmal dilatation in present at the site of the fistula (case 2).

turia. Proteinuria also was present. For dialysis access, a Goretex arteriovenous fistula had been created in his left arm. He was receiving antihypertensive therapy consisting of minoxidil, furosemide, and methyldopa.

Some 33 months prior to this admission, the patient had undergone closed needle biopsy of the left kidney, which revealed glomerulonephritis. Prebiopsy angiography had demonstrated end-stage kidney disease (Fig 4). A postbiopsy nephrogram had shown no changes, and the chest film had been normal. A loud bruit was audible over the left flank. A harsh, loud, systolic heart murmur was appreciated across the precordium. Chest ro- 


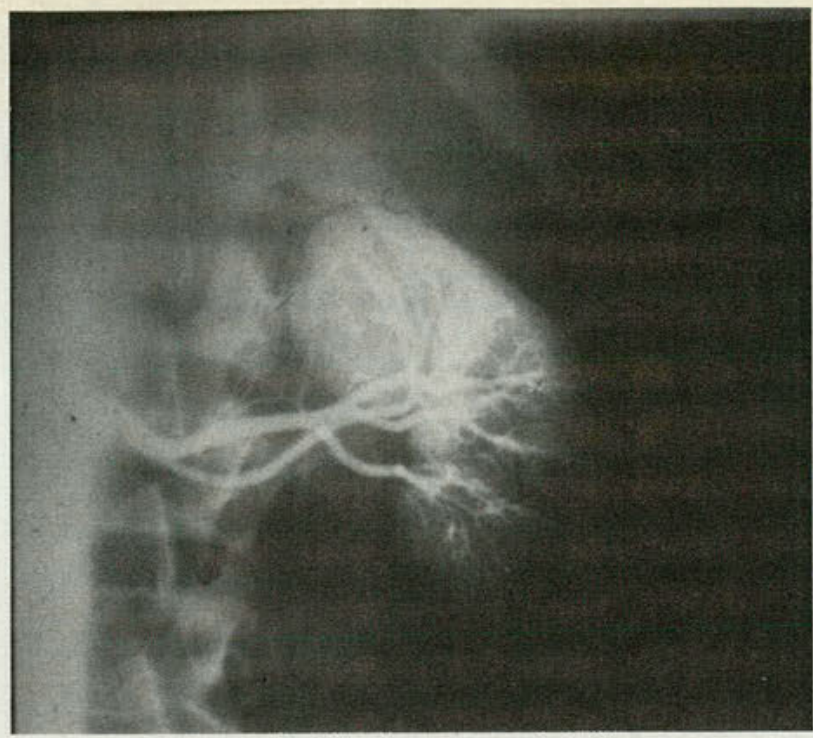

Fig 4. Angiogram of the left kidney. Pruning of the distal vessels is consistent with end-stage renal failure (case 3 ).

entgenography demonstrated cardiomegaly.

Angiography revealed a massive arteriovenous fistula with rapid emptying (Fig 5). Transcatheter intravascular occlusion of the fistula was attempted. Four Gianturco Dacron-coated, stainless-steel minicoils were injected into the fistula through the left renal artery. However, the hematuria persisted, and repeat angiography demonstrated that the fistula was still present (Fig 6).

Left nephrectomy, with individual ligation of the renal artery and vein, was performed. Prior to ligation of the artery, a thrill in the left kidney had been appreciated. Cut-section study revealed that the coils were surrounded by a thick, fibrinous tissue (Fig 7).

The postoperative course was uneventful. Furosemide was not required until discharge. No further antihypertensive medication was necessary. The patient continued to be maintained on renal dialysis following surgery and discharge. The hematuria did not reappear.

\section{Discussion}

\section{Pathophysiology}

In contrast to congenital fistulous communications, which usually are multiple, acquired fistulas seldom have more than one or two communications between the artery and vein. The fistula usually becomes apparent shortly after injury; however, a thrombus may surround the injured area and delay the diagnosis. Consequently, the capillary circulation is bypassed, and there is free flow of blood directly from the artery into the vein.

Microscopic arteriovenous channels exist in the normal vascular system under the control of the sympathetic nervous system. These shunts allow for the blood to bypass the capillary beds and are subject to chemical stimulation. Traumatic fistulas have no regulatory controls. ${ }^{2}$
The arteries and veins that communicate with the fistula undergo degenerative changes. Within the vessels, there is atrophy of the smooth muscle and elastic tissue and development of atheromatous plaques. ${ }^{3}$ The increased velocity and pressure gradients along the longitudinal axis produce elongation of the vessels. Vibratory changes (as evidenced by a thrill or bruit) result in poststenotic dilatation. ${ }^{4}$ As the vessels become dilated and tor-

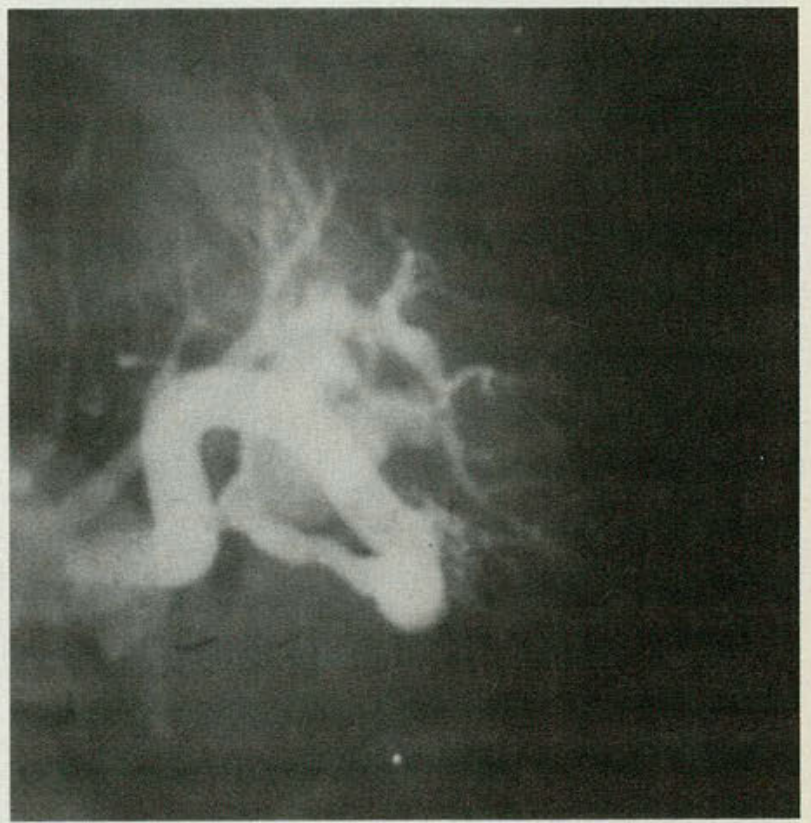

Fig 5. Angiography of the left kidney 33 months after biopsy reveals a large arteriovenous fistula (case 3 ).

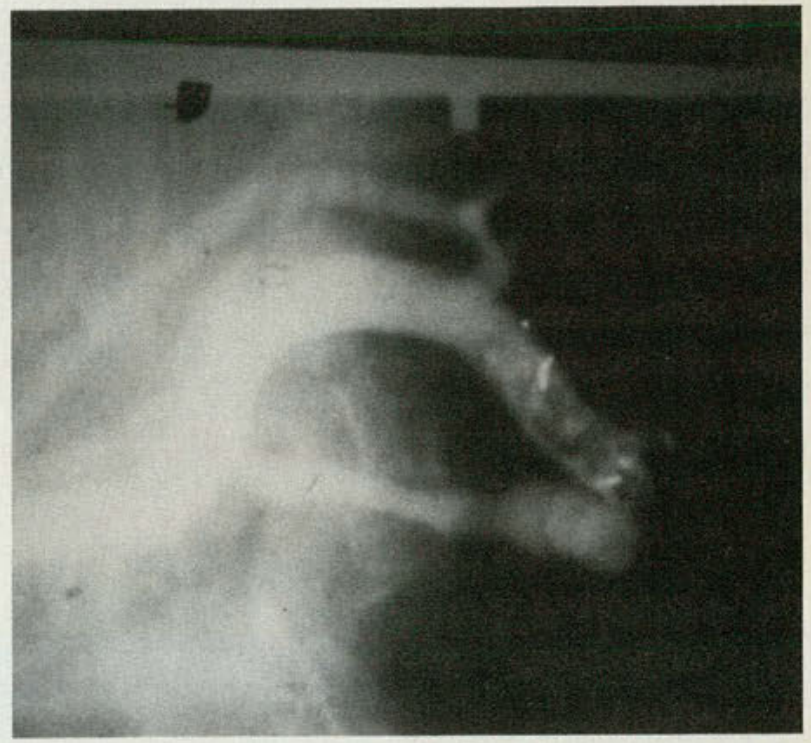

Fig 6. After the insertion of four Gianturco coils, the arteriovenous fistula persists. 


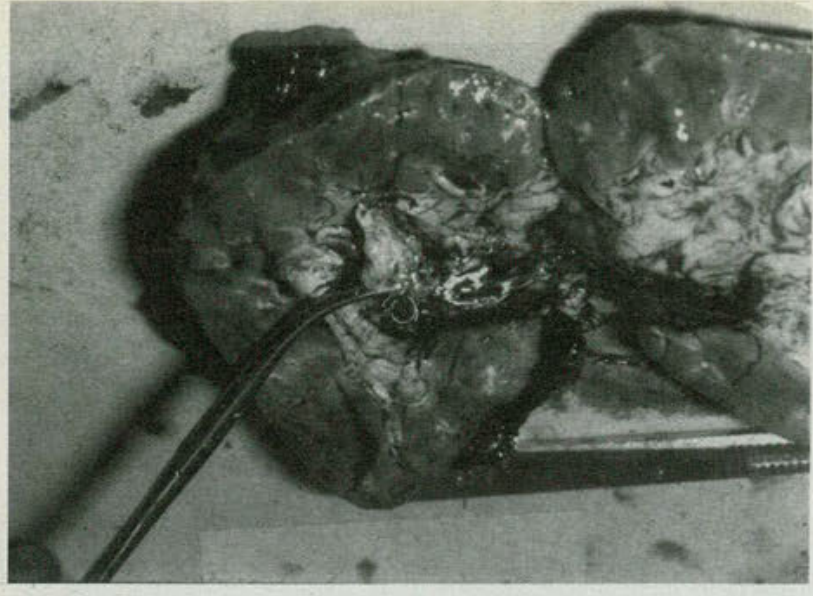

Fig 7. The cut section shows the minicoils to be embedded within the fistula in the kidney.

tuous, aneurysmal changes are not uncommon. Collateralization is enhanced, because of the increased velocity of blood flow and pressure differentials across the collateral system. ${ }^{2}$

The character of the fistulous channel itself is important. Resistance to flow is inversely proportional to both the length and diameter of the channel. Flow through the fistula increases little until its diameter approaches $20 \%$ of the diameter of the proximal artery (Fig 8). There is a rapid rise in flow as the diameter increases and resistance becomes negligible. Maximum flow occurs when the fistula's size approaches $75 \%$ of the proximal artery's diameter. The distensibility of the fistulous rim is a factor in determining the volume of blood that is diverted through the fistula. If the rim is rigid from fibrosis, the volume will be limited. ${ }^{5}$

There is a transient decrease in the pressure of the proximal artery. A compensatory increase in cardiac output results in a several-fold increase in blood flow through the proximal artery. ${ }^{6}$ Peak flow rates in the proximal vein coincide with arterial systole. The low resistance of the venous system and increased capability for dilatation allows for this greater flow at moderately low pressures. Diastolic flow also is increased-to $80 \%$ to $90 \%$ of systolic flow..$^{7-9}$ The pressure in the distal artery will usually be no greater than $60 \%$ of the systemic pressure. ${ }^{10}$ If the distal pressure is greater than the pressure in the fistula, distal flow will become retrograded.

Distal venous pressure increases. As the veins dilate, the valves no longer coapt properly and become incompetent to varying degrees. ${ }^{6,10,11}$ Flow will be diverted toward the heart through the venous collateral system. ${ }^{9}$

Flow distal to the fistula progressively decreases, and collateralization develops in both the arterial

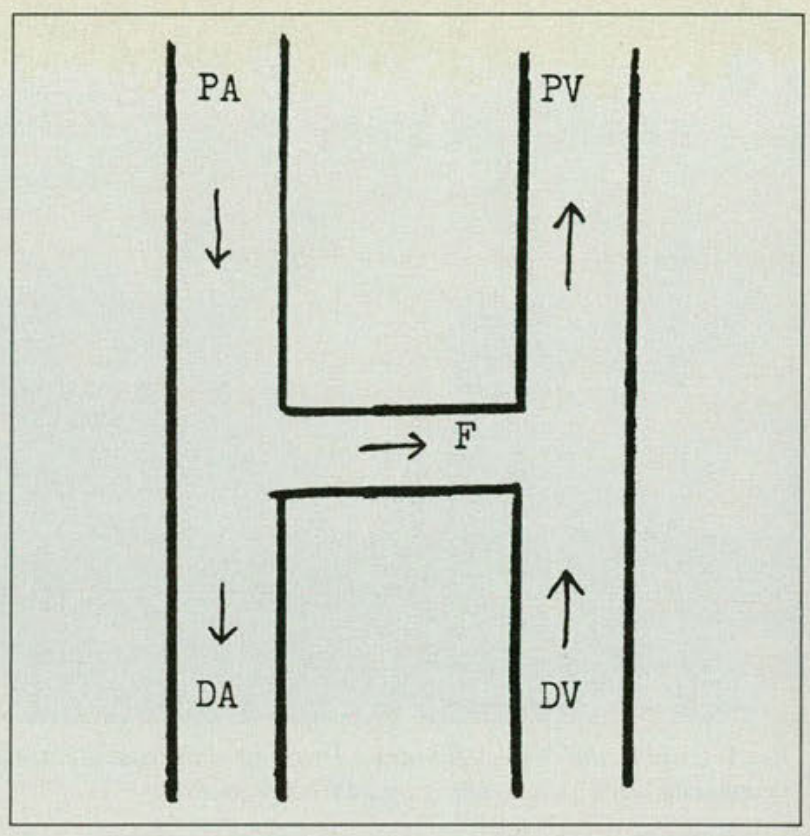

Fig 8. Early fistula development, with vascular flow still in the appropriate direction. $P A$ and $D A=$ proximal and distal arteries; $F=$ fistula ; and $P V$ and $D V=$ proximal and distal veins.

and venous systems (Fig 9). The increased size of the fistula promotes peripheral arterial and venous flow around it, but reverses the direction of arterial and venous flow distal to the fistula. The blood pressure in the distal vein approaches that of the distal artery. The retrograde flow in the distal artery (toward the fistula) decreases the outflow resistance encountered by the collateral circulation. The distal circulation is maintained by collateral inflow, but ischemia develops to varying degrees. The retrograde flow is a major stimulus for the development of arterial collateralization. ${ }^{5}$

There is an initial increase in cardiac output (CO). This is a reflection of the increased stroke volume (SV), with only a slight increase in the heart rate (HR). ${ }^{12}$ The pulse pressure (PP) widens as a result of the decreased diastolic pressure (water-hammer pulse). For the SV to increase, blood volume (BV) must increase, and venous return (VR) must improve. If the $\mathrm{CO}$ does not increase sufficiently to maintain a peripheral blood flow equal to the flow through the fistula, and if the metabolic demands of the surrounding tissues are not met, blood flow is recruited from peripheral vascular beds. ${ }^{13}$ Consequently, there usually is some degree of peripheral vasoconstriction and increased systemic vascular resistance (SVR) (Table 1).

When the fistula flow approaches $20 \%$ to $50 \%$ of the flow through the proximal artery, cardiac failure may develop if the $\mathrm{CO}$ is inadequate. This 
results from an imbalance between the increasing cardiac workload/oxygen consumption and the decreasing coronary perfusion. Cardiac hypertrophy with chamber dilatation will develop. ${ }^{12,14-16}$

Errors in diagnosis are not uncommon. The classic finding of a thrill or bruit may not be appreciated for quite some time. Frequently, the patient's symptoms are negligible or insignificant. The venous hypertension that develops is frequently misinterpreted as being secondary to varicose veins.

\section{Therapy}

Multiple indications for eradication of the arteriovenous fistula exist. Cardiopulmonary insufficiency and failure in these patients may be life threatening. Regional arterial and venous insufficiency, once present, rarely subside spontaneously. Local symptoms (pain, swelling, skin ulceration, limited extremity activities) may be incapacitating. Bacterial endocarditis and endarteritis are ever-present threats in fistulous disease.

There has been a significant decline in the incidence of traumatic arteriovenous fistulas in both military and civilian settings. Early surgical repair of arterial injuries has been responsible for the decreased numbers of delayed complications. ${ }^{17}$

The ideal therapy would be interruption of the fistula via preservation or reconstruction of the involved vessels. This would allow for the circulation to normalize. That is, once the fistula is corrected, the $\mathrm{CO}, \mathrm{HR}, \mathrm{BP}$, and $\mathrm{BV}$ return to normal. The enlarged heart shrinks, and cardiac failure is reversed.

Over the years, multiple procedures have produced less than optimal results. In the not-sodistant past, it was the custom to delay surgery for two or three months to allow for the development of collateral circulation in the area of the fistula. It was thought that primary ligation of the proximal artery could then be performed without vascular jeopardy to the distal extremity. Proximal artery ligation (per Hunter's technique) has been shown to not only decrease the peripheral blood flow by stealing blood from the collateral channels that bypass the fistula, but also that the fistula will most likely persist. ${ }^{2}$ Thus, peripheral flow into the distal artery will continue in a retrograde manner through the fistula.

Early surgical intervention has been shown not only to satisfactorily correct the fistula, but also that it will keep systemic complications to a minimum. ${ }^{18}$

Distal artery ligation will decrease the fistula flow, because the retrograde flow is eliminated. ${ }^{19}$ However, the peripheral flow will increase and permit collateral flow to reach the distal tissues. Oc-

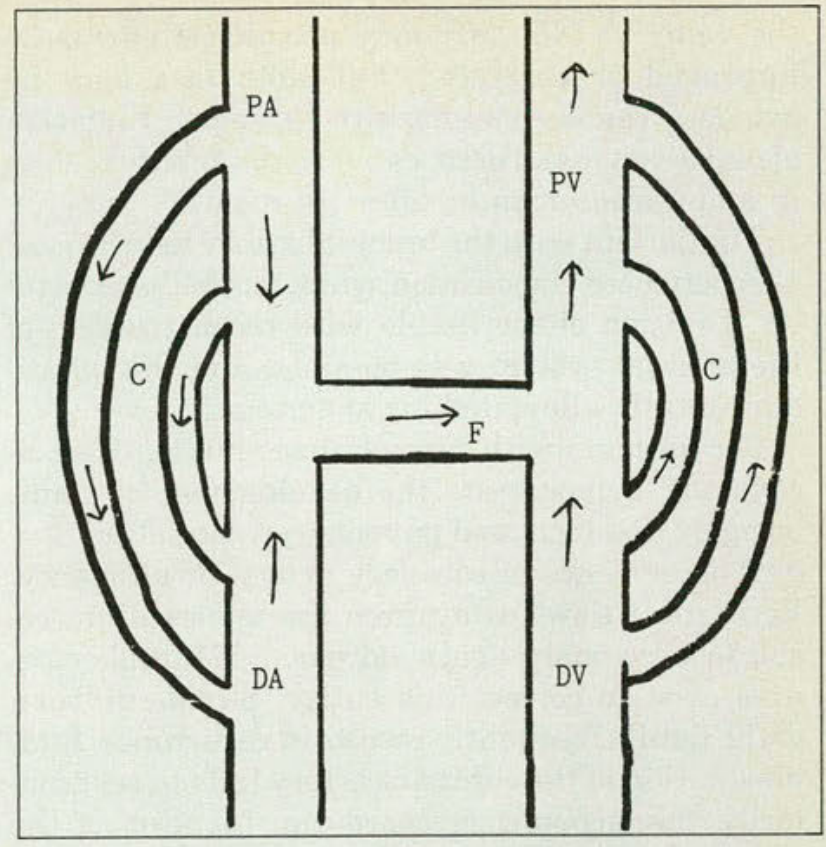

Fig 9. Advanced fistula, with extensive development of collateral vessels. The direction of the flows in the distal vessels has been reversed. This phenomenon promotes and maintains the flow through the fistula, and it may result in decreased flow in the distal organ or extremity. The diminished flows distally (and subsequent ischemia) are strong stimuli for the development of collateral circulation. $P A$ and DA = proximal and distal arteries; $F=$ fistula $; P V$ and $D V=$ proximal and distal veins; and $C=$ collateral vessels.

\begin{tabular}{|lr|}
\hline \multicolumn{2}{|l|}{ TABLE 1. SYSTEMIC MANIFESTATIONS OF ARTERIOVENOUS } \\
FISTULAS. & Change \\
\hline Parameter & +++ \\
\hline Cardiac output (CO) & ++ \\
Stroke volume (SV) & + \\
Heart rate (HR) & ++ \\
Pulse pressure (PP) & ++ \\
Blood volume (BV) & ++ \\
Venous return (VR) & + \\
Systemic vascular resistance (SVR) & \\
\end{tabular}

clusion of both the proximal and distal veins will increase the resistance through the fistula, with subsequent increase in peripheral and collateral perfusion. ${ }^{5}$

Quadruple ligation, which initially was performed by Bramann, ${ }^{2}$ is physiologically sound. Ligation of all four vessels eliminates the fistula and allows the collateral vessels to maintain viability of the distal tissues. ${ }^{20}$ However, arterial insufficiency becomes apparent $40 \%$ to $50 \%$ of the time. ${ }^{2}$

Arterial surgical reconstruction, either via bypass or arteriorrhaphy, has been shown to be the clinically superior procedure. As the distal tissues become revascularized, the metabolic demands are satisfied. Attempts should also be made to preserve 
the vein. ${ }^{20-22}$ Not only may peripheral edema be improved or corrected, but limb loss may be avoided. Venous trauma with subsequent ligation of major venous structures not uncommonly results in amputation months after the event. ${ }^{20}$

The patient with the brachial artery fistula (case 1) experienced claudication symptoms in the extremity. Division of the fistula with reconstruction of the arterial system was possible, and this procedure totally alleviated his symptoms.

For patients with intracardiac shunts, chest xrays will demonstrate the development of cardiomegaly and increased pulmonary vasculature. Angina is evidence of coronary artery insufficiency. Retrograde flows away from the myocardium result in a "coronary steal syndrome." 23 Multiple meaures exist to correct this entity. Simple division of the fistula frequently results in recurrence. Simple ligation of the coronary artery fails to revascularize the ischemic myocardium. Ligation of the artery above and below the fistula with distal bypass is the recommended treatment of choice. ${ }^{24}$

Noninvasive measures to obliterate fistulas when successful, usually result in sustained fistula occlusion. Embolization therapy may be performed percutaneously using autologous clot, Gelfoam, Silastic catheters, muscle, wire, or coils. Needle biopsy of the kidney occasionally results in a small fistula, which usually resolves spontaneously. Definitive care is indicated if the fistula persists for longer than six months, if hematuria or flank pain develops, if hypertension becomes increasingly difficult to control, or if symptoms of cardiac failure develop. Preservation of a poorly functioning kidney was not indicated in case 3 .

\section{Summary}

Traumatic arteriovenous fistula results from simultaneous injury to an adjacent artery and vein. Most small fistulas remain asymptomatic and resolve spontaneously. Larger fistulas, with pressures that approach the pressure of the proximal artery, produce both local and systemic symptoms. Ischemic symptoms develop in tissues distal to the fistula. Cardiac hypertrophy and pulmonary hypertension result from a sustained increase in cardiac output and blood volume. Respiratory symptoms are not uncommon.

Correction of the arteriovenous fistula should be performed when the local symptoms become troublesome or incapacitating, and prior to the development of cardiopulmonary insufficiency. An aggressive approach is indicated. Angiography is necessary not only to confirm the diagnosis, but to help to coordinate corrective therapy. Preservation of the arterial circulation to the distal tissues pro- duces optimal results. Attempts should also be made to preserve the venous structures.

1. Rich NM, Hobson RW II, Collins GJ: Traumatic arteriovenous fistulas and false aneurysms: A review of 558 lesions. Surgery 1975;78:817. 828.

2. Rutherford, RB (ed): Vascular Surgery, ed 2. Philadelphia, WB Saunders, 1984.

3. Stehbens WE, Karmody AM: Venous atherosclerosis associated with arteriovenous fistulas for hemodialysis. Arch Surg 1975;110:176-180.

4. Rodbard S, Ikeda K, Montes M: An analysis of mechanisms of poststenotic dilatation. Angiology 1967;18:349-369.

5. Wilson SE, Owen L: Vascular Access Surgery. Chicago, Year Book Medical Publishers, Inc, 1980.

6. Strandess DE, Summer DS: Hemodynamics For Surgeons. New York, Grune \& Stratton, Inc, 1975.

7. Lavigne JE, Brown CS, Fewel J, et al: Hemodynamics within a canine femoral arteriovenous fistula. Surgery 1975;77:439-443.

8. Schenk WG, Bahn RA, Cordell AR, et al: The regional hemodynamics of experimental acute arteriovenous fistula. Surg Gynecol Obstet 1957;105:733-740.

9. Schenk WG, Martin JW, Leslie MB, et al: The regional hemodynamics of chronic experimental arteriovenous fistula. Surg Gynecol Obstet $1960 ; 110: 44-50$.

10. Hobson RW II, Croom RD III, Swan KG; Hemodynamics of the distal arteriovenous fistula in venous reconstruction. J Surg Res 1973;14:483489.

11. Hol R, Ingebrigtsen R: Experimental arteriovenous fistulae. Acta Radiol 1961;55:337-349.

12. Johnson G Jr, Blythe WB: Hemodynamic effects of arteriovenous shunts used for hemodialysis. Ann Surg 1970;171:715-732.

13. Leslie MB, Portin BA, Schenk WG: Cardiac output and posture studies in chronic experimental arteriovenous fistulas. Arch Surg 1960;81:123128

14. Anderson CB, Etheredge EE, Harter HR, et al: Blood flow measurements in arteriovenous dialysis fistulas. Surgery 1977;81:459-461.

15. Crowe CP, Schenk WG: Massive experimental arteriovenous fistulas. J Trauma 1963;3:13-21.

16. Rowe GG, Castillo CA, Afonso S, et al: The systemic and coronary hemodynamic effects of arteriovenous fistulas. Am Heart $J$ 1962;64:44 49.

17. Drapanas T, Hewitt RL, Weichert RF III, et al; Civilian vascular injuries: A critical appraisal of three decades of management. Ann Surg 1970;172:351-360.

18. Johnson G: Local pathophysiology of an arteriovenous fistula, in Swan KG (ed): Venous Surgery in the Lower Extremity. St. Louis, Warren H Green, Inc, 1976 .

19. Spencer FC, Grewe RV; Management of arterial injuries in battle casualties. Ann Surg 1955;141:304-313.

20. Rich NM, Hughes CW, Baugh JH: Management of venous injuries. Ann Surg 1970;171:724-730.

21. Hughes CW, Jahnke EJ: The surgery of traumatic arteriovenous fistulas and aneurysms: A five-year follow-up study of 215 lesions. Ann Surg 1958;148:790-797.

22. Hewitt RL, Smith AD, Drapanas T: Acute traumatic arteriovenous fistulas. J Trauma 1973;13:901-906.

23. Rowe GG: Inequalities of myocardial perfusion in coronary artery disease ("coronary steal"). Circulation 1970;42:193-194.

24. Haas GE, Parr GV, Trout RG, et al: Traumatic coronary artery fistula. J Trauma 1986;26:854-857.

Special acknowledgement to Drs R.S. Swan, G. Paar, R.G. Trout, and the Presbyterian University of Pennsylvania Medical Center and Metropolitan Hospital, Philadelphia, for their assistance.

Dr Haas is in private practice of peripheral vascular and cardiothoracic surgery in Langhorne, Penn.

Reprint requests to Dr Haas, 400 Middletown Blvd, Suite 112, Langhorne, PA 19047. 